\title{
Electro-assisted Deposition of Calcium Phosphate on Self-Assembled Monolayers
}

\author{
Noah Metoki, ${ }^{a}$ Kazi Sadman, ${ }^{\mathrm{b}}$ Kenneth Shull, ${ }^{\mathrm{b} *}$ Noam Eliaz ${ }^{\mathrm{a}, *}$ and Daniel Mandler ${ }^{\mathrm{c}, *}$ \\ ${ }^{a}$ Biomaterials and Corrosion Lab, Department of Materials Science and Engineering, Tel- \\ Aviv University, Ramat Aviv 6997801, Israel \\ b Polymer Surfaces \& Interfaces Lab, Department of Materials Science and Engineering, \\ Northwestern University, Evanston, IL 60208, USA \\ ${ }^{c}$ Institute of Chemistry, The Hebrew University of Jerusalem, Jerusalem 9190401, Israel
}

\begin{abstract}
Calcium phosphate $(\mathrm{CaP})$ ceramics are used in orthopedics and dentistry due to their excellent biocompatibility and osseointegration. Here, the electro-assisted deposition of CaP on two different self-assembled monolayers (SAMs), 2-mercaptoacetic acid (MAA) and 2mercaptoethanol (ME), were studied both at short (up to $3 \mathrm{~min}$ ) and at long (2 hours) deposition periods on well-defined evaporated gold surfaces. It was found that the end group of the monolayer has a major effect on the growth of the $\mathrm{CaP}$ coating. The deposition was slower and less electrically efficient on MAA SAM, but surface cracking was essentially eliminated due to reduction of the crystallographic mismatch. The carboxylic acid may facilitate $\mathrm{CaP}$ growth by attracting $\mathrm{Ca}^{2+}$ ions to the surface, which could explain the higher amount of side reactions occurring at the beginning of the deposition.
\end{abstract}

Keywords: Calcium phosphate (CaP), hydroxyapatite (HAp), electrodeposition, self-assembled monolayers (SAMs).

Corresponding author address: E-mail: daniel.mandler@mail.huji.ac.il; Tel: +972 2 6585831; Fax: +97226585319 . 


\section{Introduction}

Calcium phosphate $(\mathrm{CaP})$ is the major component of the bone. It represents a family of materials consisted of various phases, among others, hydroxyapatite (HAp), $\alpha$ - and $\beta$ tricalcium phosphate (TCP), and octacalcium phosphate (OCP) [1]. One of the major challenges in biomaterial research during the last decades has been implant design as a means of accelerating post-surgery bone healing. As a result, several methods have been developed for coating implants with $\mathrm{CaP}$ in order to enhance their fixation. Since the Food and Drug Administration (FDA) approved CaP-based cements containing specifically HAp, OCP and TCP for human use in the 1990s, these phases have become prevalent in coatings as well [2]. HAp is especially popular since it bears the greatest similarity to biological apatite, both in crystallographic structure and $\mathrm{Ca} / \mathrm{P}$ ratio [3].

Today, plasma spraying (PS) is the most common technology used commercially for coating implants with $\mathrm{CaP}$. Since the early 1990s, however, much interest in electrodeposition of $\mathrm{CaP}$ has evolved due to the advantages it offers, such as non-line-of-sight coating and lower residual stresses [4]. The electrodeposition of $\mathrm{CaP}$ from aqueous solution is driven by cathodic potential, which causes the elevation of $\mathrm{pH}$ and thus the deprotonation of phosphate species to form insoluble $\mathrm{CaP}$ [3]. This process has been studied mostly on reactive metals, primarily on titanium and its alloys. For example, Gopi et al. [5] studied the film composition of electrodeposited $\mathrm{CaP}$ on stainless steel. They suggested that in acidic aqueous solution containing calcium and phosphate ions, the electrochemical and chemical reactions that follow the deprotonation of phosphate, first yield the deposition of dicalcium phosphate dihydrate (Brushite, DCPD) while OCP is deposited more slowly. This was then reversed when the $\mathrm{pH}$ increased. Montero-Ocampo et al. [6] studied the $\mathrm{CaP}$ coating on $\mathrm{Ti}$ alloy at varied controlled potentials. They showed that the kinetics of CaP precipitation was enhanced by sufficiently negative potentials. The latter determined the structure of the 
coating. Eliaz et al. studied the effect of surface mechanical and chemical pre-treatments on electrodeposited $\mathrm{CaP}$. It was shown that the current transient during deposition was affected by the surface pre-treatment, consequently resulting in different surface morphologies of the coating [7].

The electrocrystallization of $\mathrm{CaP}$ on titanium has also been studied. Eliaz et al. [3] investigated the nucleation of $\mathrm{CaP}$ on titanium and concluded that it involved two stages: 2D instantaneous nucleation followed by $3 \mathrm{D}$ progressive nucleation. Nucleation commenced at $-0.84 \mathrm{~V}$ (vs. SCE) while potentials that were more negative than $-1.26 \mathrm{~V}$ were required for enhanced growth. Mass transport was found to have only secondary effect on the deposition process. Grubač et al. [8] concluded based on fitting of the current-time transients to various nucleation models that instantaneous 3D nucleation under diffusion control took place. Metikoš-Huković et al. [9] showed that the chronoamperometry (CA) of $\mathrm{CaHPO}_{4}$ nucleation on Mg-alloy deviated from 3D instantaneous nucleation, while its nucleation on glassy carbon followed progressive 3D nucleation.

As described earlier, the course of crystal growth reactions and the properties of the electrodeposits are markedly influenced by many parameters, such as surface pretreatment, bath composition, oxygen level, $\mathrm{pH}$, stirring, the applied potential (or current density), temperature, and cell geometry. In addition, defects on the surface play a major role in the nucleation and growth of electrodeposits [3]. Namely, in order to thoroughly investigate the growth and nucleation process, it is essential to work with well-defined and reproducible surfaces. The latter are relatively easy to form by self-assembled monolayers (SAMs).

It has been shown that surfaces modified by SAMs bearing various functional endgroups had a significant effect on the chemical precipitation and nucleation of CaP [10-13]. Ordinarily, the end-group of the monolayer was chosen to mimic the biomineralization process in the body, where the growth of HAp is associated with the organization of the 
collagen fibers. Thus, it is believed that interfacial interactions between HAp and the functional groups on the side-chains of the collagen molecules are crucial in this process. Accordingly, various SAMs terminated with hydroxyl $(\mathrm{OH})$, sulfonic acid $\left(\mathrm{SO}_{3} \mathrm{H}\right)$, phosphate $\left(\mathrm{PO}_{4} \mathrm{H}_{2}\right)$ and carboxylic acid $\left(\mathrm{CO}_{2} \mathrm{H}\right)$ have been studied [10-13]. Some of the negatively charged end-groups were found effective in promoting the mineralization process from solution and affected the texture of the CaP coating [13]. Yet, there is an active discussion in the literature regarding which SAM is most beneficial. To the best of our knowledge, SAMs have never been studied in the context of CaP electrodeposition.

The objective of this work is to investigate $\mathrm{CaP}$ nucleation and growth on welldefined surfaces. Specifically, the electrodeposition of $\mathrm{CaP}$ was studied both on bare gold and on gold surfaces covered with 2-mercaptoacetic acid (MAA) and 2-mercaptoethanol (ME) SAMs. We found that the presence of ME has only a minor effect on the adhesion of the deposit. On the other hand, MAA SAM affects not only the nucleation, but also the growth rate of the coating and its final surface morphology.

\section{Experimental}

\subsection{Chemicals}

2-mercaptoacetic acid (MAA, 98\%), 2-mercaptoethanol (ME, 98\%), analytical grade sulfuric acid, calcium nitrate (99\%), ammonium dihydrogen phosphate (98\%), analytical grade sodium hydroxide, analytical grade hydrochloric acid, analytical grade sodium chloride, reagent grade sodium phosphate monobasic, and reagent grade calcium chloride anhydrous were purchased from Sigma-Aldrich. Deionized (DI) water $(>18 \mathrm{M} \Omega \cdot \mathrm{cm})$ was used for all the experiments.

\subsection{Instruments and programs}


Electrochemical deposition (ED), chronoamperometry (CA) and cyclic voltammetry (CV) were carried out with a CHI-750B potentiostat (CH Instruments Inc., TX, USA). Electrochemical quartz crystal microbalance (EQCM, Advanced Wave Sensors, Valencia, Spain) was connected to a network analyzer (250 B/C, Saunders and Associates) and a potentiostat (BioLogic SP-150, France).

X-ray photoelectron spectroscopy (XPS) spectra were recorded using an Axis Ultra spectrometer (Kratos) and $\mathrm{Mg}-\mathrm{K} \alpha$ radiation of $1486.71 \mathrm{eV}$. Data were collected and analyzed by a Vision processing program. The surface morphology was analyzed by an environmental scanning electron microscope (ESEM, Quanta 200 FEG, FEI).

The saturation index (SI) was calculated for the different $\mathrm{CaP}$ phases using PHREEQC computational program. The procedure of such calculations is described elsewhere in detail [1].

\subsection{Procedures}

Preparation of the gold samples: evaporated samples of gold $(15 \mathrm{~nm} \mathrm{Cr}+100 \mathrm{~nm} \mathrm{Au})$ on glass, $1.5 \mathrm{~cm}^{2}$ in area, were used for ED. An AT-cut (Inficon, East Syracuse, NY, 1.27 $\mathrm{cm}^{2}$ ), $5 \mathrm{MHz}$ Au-coated quartz crystals were used for EQCM experiment. The samples were treated for $15 \mathrm{~min}$ in a UV/ozone apparatus (UVOCS, Philadelphia, US). They were then immediately immersed in $1 \mathrm{mM}$ solution of either MAA or ME in ethanol overnight. This was followed by dipping the samples three times in DI water at ambient temperature. Evaporated gold samples for ED were annealed after the UV/ozone cleaner using a torch. CA experiments utilized a gold disc ( $2 \mathrm{~mm}$ in diameter) that was pretreated by electrocycling the electrode using $\mathrm{CV}$. This was performed in $0.5 \mathrm{M}$ sulfuric acid, from $-0.5 \mathrm{~V}$ to $1.6 \mathrm{~V}$, at a scan rate of $0.5 \mathrm{~V} \mathrm{~s}^{-1}$, for 40 cycles.

Electrochemical cell: ED was carried out in a standard three-electrode cell $(15 \mathrm{~mL})$ in which platinum wire and an $\mathrm{Ag} / \mathrm{AgCl}(3 \mathrm{M} \mathrm{KCl})$ electrode were used as the counter and 
reference electrodes, respectively. Therefore, all potentials are given hereafter $v s$. this reference electrode.

EQCM: Prior to deposition of $\mathrm{CaP}$, the entire EQCM cell was placed in a $37^{\circ} \mathrm{C}$ water bath and allowed to equilibrate. The resonance frequency of the bare crystal in solution was then measured for 10 minutes, and the subsequent frequency shifts during CaP ED were referenced to the bare crystal in solution. Deposition was carried out in a three-electrode cell with the working electrode set at $-1.0 \mathrm{~V}$ for two hours. A platinum counter electrode was used.

The electrolyte for deposition in both EQCM and $\mathrm{ED}$ was $0.61 \mathrm{mM} \mathrm{Ca}\left(\mathrm{NO}_{3}\right)_{2}+0.36$ $\mathrm{mM}\left(\mathrm{NH}_{4}\right) \mathrm{H}_{2} \mathrm{PO}_{4}$ in DI water. The $\mathrm{pH}$ of the bath was measured using an InoLab $\mathrm{pH} / \mathrm{Oxi}$ Level 3 meter, and adjusted to $\mathrm{pH} 7.4$ by addition of $\mathrm{NaOH}$ before the experiment started. The bath temperature was kept at $37 \pm 0.1{ }^{\circ} \mathrm{C}$ by means of a hot plate with temperature control. ED was carried out by applying a constant potential of $-1.36 \mathrm{~V}$ for 2 hours. After ED, the samples were washed in DI water. Finally, the samples were dried and stored in a Petri dish sealed in a polyethylene bag.

CA: After cleaning, the samples were immersed in $1 \mathrm{mM}$ thiol solution in ethanol for one hour. The samples were carefully washed before transferring into the electrochemical cell. The current transient under constant potential of $-1.36 \mathrm{~V}$ was recorded in solutions containing $0.1 \mathrm{M} \mathrm{NaCl}$ ("Blank") and $0.1 \mathrm{M} \mathrm{NaCl}$ containing $0.61 \mathrm{mM}$ calcium chloride and $0.36 \mathrm{mM}$ sodium phosphate monobasic ("Low") at constant $\mathrm{pH}$ 7.4. Nitrate ions were eliminated in these CA experiments to avoid their contribution to the formation of hydroxyl ions. The solution was bubbled with argon for $10 \mathrm{~min}$ prior to the experiment.

$\mathrm{CV}$ : After cleaning, the samples were immersed in the deposition solution. The CV was carried out at a scan rate of $10 \mathrm{mV} \mathrm{s}^{-1}$, from 0 to $-1.5 \mathrm{~V}$ and back. 


\section{Results and Discussion}

The electrochemical deposition (ED) of $\mathrm{CaP}$ is based on altering the $\mathrm{pH}$ in the vicinity of the electrode surface as a result of applying a negative potential. The $\mathrm{pH}$ change shifts the equilibrium into an unstable state where $\mathrm{CaP}$ precipitates. Therefore, the first step in this work involved calculation and characterization of the solutions used for ED. The saturation index (SI, eq. 1) is a measure of saturation defined as the ratio between the ion activity product in non-equilibrium, Q, and the thermodynamic solubility product, $\mathrm{K}_{\mathrm{SP}}$. Clearly, SI is larger than zero for supersaturated solutions and smaller for undersaturation. SI was calculated for the different CaP phases using PHREEQC computational program.

$$
\text { (1) } \quad \mathrm{SI}=\log _{10}\left(\frac{Q}{K_{S p}}\right)
$$

Figure 1 shows the calculated SI for the solution used for ED. It can be seen that throughout the entire $\mathrm{pH}$ range the solution is supersaturated with respect to HAp. Furthermore, under the initial conditions, i.e. $\mathrm{pH} 7.4$, tricalcium phosphate (TCP) is also at supersaturation conditions. Increasing the alkalinity by one $\mathrm{pH}$ unit causes octacalcium phosphate (OCP) and diabasic calcium phosphate anhydrate (Monetite, DCPA) to oversaturate, which can, in principle, result in their precipitation.

It is known that $\mathrm{CaP}$ can form suspended-supersaturated solutions that are metastable $[14,15]$. In this solution, precipitation does not take place spontaneously at short durations due to a kinetic barrier, which can be surpassed by applying energy or by increasing the $\mathrm{pH}$, vide infra. This solution is clear and represents such a case.

Based on our previous experience, the $\mathrm{ED}$ of $\mathrm{CaP}$ can be driven by shifting the $\mathrm{pH}$ $[1,3,16]$. Hence, we examined the CV of the solution used for ED in order to determine at which potential the reduction of water commences. Figure 2 shows the CV recorded with either bare Au electrode or Au electrodes coated with SAMs. It can be seen that the reduction of water begins at ca. $-1.1 \mathrm{~V}$ for both the bare and the ME-coated electrodes, whereas it is 
slightly shifted to more negative potentials (by ca. $0.1 \mathrm{~V}$ ) for the MAA-coated electrode. This is likely to affect the deposition of $\mathrm{CaP}$, as will be shown later. The increase of the current (without obtaining a peak) is expected for the reduction of the solvent.

It should be noted that the negative potential might affect, and even cause, the reductive desorption of the SAMs. It was reported that thiols can be reduced at potentials of $-1.0 \pm 0.25 \mathrm{~V}$ [17]. This has been intensively studied and usually conducted under very basic conditions, i.e. $0.5 \mathrm{M} \mathrm{KOH} \mathrm{[18].}$

We did not observe any electroreduction of the thiols, and the results were highly reproducible. Yet, to confirm that the thiols were not reduced under the experimental conditions, XPS analysis of the surface was done before and after applying similar negative potential. It was ensured that the solution contained only phosphate and was devoid of calcium thereby preventing a precipitation. From the analysis, we found that the thiols remained on the surface.

The next step in studying the electrochemical deposition of $\mathrm{CaP}$ on $\mathrm{Au}$ and SAMscovered Au was by $\mathrm{CA}$. The early stages of nucleation and growth are often examined by following the current transient as they reflect the processes occurring at the electrode/electrolyte interface. Nevertheless, in our case the deposition is driven indirectly by potential; the latter does not cause the reduction or oxidation of the deposit precursor as in electroplating of metals [3]. Therefore, it is not obvious that the deposition will affect the CA. It should be noted that the time scale of CA can be relatively long, as has been shown by Eliaz et al. [3], because the build-up of a $\mathrm{pH}$ gradient is required to drive the deposition. At the same time, Metikoš-Huković et al. [9] reported the CA of magnesium alloy and glassy carbon on a short time scale of ca. $0.1 \mathrm{~s}$.

Figure 3 shows the CA of a bare gold electrode (A), Au covered by MAA and ME (B and $\mathrm{C}$, respectively), and a comparison between the different substrates (D). The transients 
were recorded in two different solutions: 1) "Blank" solution containing $0.1 \mathrm{M}$ of $\mathrm{NaCl}$, and 2) "Low" solution containing calcium and phosphate concentrations as in the ED solution, and with $0.1 \mathrm{M}$ of $\mathrm{NaCl}$. Both solutions were adjusted to $\mathrm{pH} 7.4$. It can be seen that in all three cases the initial charging current decays rapidly and attains a steady-state current within a few seconds. Yet, there is a distinct difference between the "Blank" and the "Low" solutions. In all cases, when calcium and phosphate are present in solution - the cathodic current elevates.

Recalling that the major contribution to the current is the reduction of water, one may suggest that the higher current must be due to an increase of the rate of water electroreduction following the consumption of $\mathrm{OH}^{-}$ions by the calcium and phosphate ions [3]. The latter implies that the hydroxyl ions deprotonate the major phosphate species, i.e. $\mathrm{HPO}_{4}{ }^{2-}$ shifting the reduction of water. The effect of the SAMs on the CA in the "Low" solution is evident in Fig. 3D. While the CA recorded in the presence of ME SAMs shows similar behavior to that of the bare surface, the currents recorded in the presence of MAA are higher. We repeated these experiments several times to assure this difference between the bare and the MAAcoated Au. The increased cathodic current must be due to either increased electron transfer rate constant or increased concentrations of the reactants (providing that the temperature, surface area of the cathode and the applied potential are kept constant). The only conclusion that we can draw at this stage is that it is possible that the amount of side reactions occurring on top of the MAA is higher than that on the other surfaces, thus establishing higher current. This may be attributed to the acidity of the interface marked by $\mathrm{pK}_{\mathrm{a}, \mathrm{s}}$. Namely, the dissociation constant of the hydroxyl group, $\mathrm{pK}_{\mathrm{a}, \mathrm{s}}$ of $\mathrm{ME}$ is 9.64 , vs. that of the carboxyl group, $\mathrm{pK}_{\mathrm{a}, \mathrm{s}}$ of MAA is $3.83[19,20]$. It is well known that the ionic strength of the solution, roughness of the surface, chain length, etc. influence $\mathrm{pK}_{\mathrm{a}, \mathrm{s}}$ values [21-23]. Yet, most importantly in our case, the electric field can affect the protonation state of the acids localized 
at the electrode surface. This is attributed to the shift in the $\mathrm{pH}$ upon applying a potential. In our system, the carboxylic acid releases protons into solution at lower $\mathrm{pH}$, which enhances side reactions.

In order to characterize the nucleation and growth of $\mathrm{CaP}$, an EQCM was used to monitor the mass change and quantify the effect of SAMs on the deposition kinetics. If the film on the quartz surface is sufficiently rigid, then the Sauerbrey equation $[24,25]$ provides an accurate measurement of the change of mass on the crystal. In the ED of CaP, the dissipation shifts of the resonance peaks remained small relative to the frequency shifts, implying that the films lacked viscoelastic character and that the Sauerbrey condition was met. Dissipation shifts are proportional to the viscoelastic character of the deposited film and lead to deviations from the Sauerbrey condition [26]. The cathodic potential of the working electrode was set to be $-1.0 \mathrm{~V}$ in order to mitigate the formation of hydrogen bubbles $\left(\mathrm{H}_{2}\right)$ on the electrode surface, which could interfere with the EQCM response. Under the specific experimental conditions selected here, no $\mathrm{H}_{2}$ nucleation was observed during the deposition. The cathodic current density, charge density and mass calculated from the third harmonic are shown in Fig. 4. For clarity, only data from the third harmonic are presented, but there was good agreement between all the measured harmonics $(n=1,3$ and 5).

The experiments were repeated three times for the bare and modified surfaces and the results were reproducible. The currents decay to almost the same current density. More information can be extracted from the charge density transients (Figure 4B). The latter are characterized by two linear regimes that are more pronounced in the bare and the ME cases. The slope of the first regime is steeper than that of the second regime, which commences after ca. $20 \mathrm{~min}$. Figure $4 \mathrm{C}$ shows the change of the mass density, derived from the change in frequency, as a function of time. A change in the deposition density can also be seen at ca. 20 min that is in accordance with the charge transient. This suggests that the current and charge 
density can be correlated with the mass density or, in other words, the current and charge due to a Faradaic process reflect the deposition process. This means that deposition proceeds through two different periods: the first stage which takes approximately 20 min during which the growth is faster than during the second, steady-state stage.

In order to evaluate the ratio of deposit to energy invested in the system while at steady state, the slopes of both Q-t and m-t can be compared. It is evident that at steady-state the bare and $\mathrm{OH}$-covered surfaces are characterized by similar deposition rate and charge transfer rate whereas the mass per charge on the MAA-covered surface is lower by half. Furthermore, when dividing $\mathrm{Q}$ by the Faraday constant and the accumulated mass by the molar mass (of the OCP phase, as will be shown later), a mole-per-mole graph is drawn (Fig. 4D). This graph clearly shows the low efficiency of the coating process. It is required to pass $\sim 500$ nmoles of electrons in order to deposit 20 nmoles of CaP. The deposition on the bare and the ME-coated electrodes behaves similarly, with two distinct growth periods. The second period in these samples is more efficient, and implies the growth of the coating. On the other hand, the deposition on the MAA-covered surface is, in general, less efficient and remains more or less constant.

For comparison, Eliaz and Eliyahu [3] used electrochemical atomic force microscopy (EC-AFM) to study the electrochemical processes of nucleation and growth of hydroxyapatite on titanium. Based on normalized current transients, atomic force microscope (AFM) deflection images, and comparison to ESEM and transmission electron microscope (TEM) images it was concluded that during the first 12 min of deposition, the nucleation is instantaneous and is accompanied by two-dimensional (2D) growth. Subsequently, the nucleation becomes progressive and is accompanied by a three-dimensional (3D) growth. In the case of biomineralization in vivo too, the average crystal size is smallest at formation and increases to maturity, at which time there is a leveling off of this growth process. Eliaz et al. 
[25] subsequently showed, using real-time EQCM measurements and advanced XPS analysis, that an incubation time of $\sim 11 \mathrm{~min}$ is required for local increase of $\mathrm{pH}$ due to electrolysis of water before precipitation from solution can occur. It was also argued that the low solubility product allows precipitation of $\mathrm{CaP}$ even at relatively low concentrations of calcium and phosphate/hydrogen phosphate ions in solution, and that HAp most likely forms via transformation of precursor phases, such as OCP, and not directly.

Here, more insights on the nucleation and growth were obtained by SEM (Fig. 5). Different substrates, i.e., bare gold and gold coated with MAA, were subjected to a potential of $-1.36 \mathrm{~V}$ for $30 \mathrm{~s}, 1,2$ and 3 minutes. Due to major similarities in the characteristics of bare and ME-coated substrates, a comparison is made here only between the bare and MAAcoated substrates. It can be seen that $\mathrm{CaP}$ grows on the MAA-covered samples in a very different manner than on the bare samples. The latter hardly show any change as compared to the surfaces not coated with $\mathrm{CaP}$ in the first $30 \mathrm{~s}$ (Supplementary Information, Figure 1). At larger magnifications, however, a coating can be seen. The Au surface coated with SAMs looks very different after applying a negative potential for $30 \mathrm{~s}$. The formation of "islands" is visible. An examination of the surface under backscattered electrons (BSE) reveals that the change in the contrast does not stem from compositional changes, but only from topographical changes. The growth of these islands until covering the whole surface is seen up to 2 min, after which the surface becomes uniform. The bare surface, on the other hand, shows a non-uniform coating. The white dots detected as a heavier material in highmagnification BSE images are attributed to the gold surface.

These data suggest that the change of end group is substantial in influencing the growth of the coating. Uchida et al. [27] discovered that the epitaxial relationship between the substrate and apatite may lead to favorable apatite formation on different substrates. Moreover, better matching between the crystallographic planes, even by $1 \%$, will achieve 
favorable growth. The fact that the growth on the bare substrate is non-uniform is not surprising, as the crystallographic structure of the gold substrate (cubic) does not match that of the coating (hexagonal or triclinic).

Comparing the bare and MAA-covered gold, the growth processes are apparently different. This is because nucleation and growth of a coating depends on the interfacial energy barrier, which is the difference between the free energy of the molecule in the bulk and at the surface [28]. Each system bears its own method of free energy minimization depending on functionalization of the substrate surface and lattice matching [29], which are both changed by the SAMs. It can be seen that $\mathrm{CaP}$ on the MAA-covered surface apparently grows in 2D layers, as oppose to the bare surface on which the growth is apparently 3D.

Further knowledge about the phase content can be gained by XPS. The phase content in CaP coatings on both bare and MAA-covered gold samples was analyzed by XPS after 3 minutes and 2 hours of deposition. Low-resolution XPS survey spectra of the surface of all samples (three points per each sample) revealed the presence of calcium, phosphorous and oxygen. Carbon was also detected after both time periods of deposition, and is likely to be the outcome of carbonate contaminations commonly found in electrochemical cells having graphite as an anode.

The atomic concentrations of elements obtained from high-resolution XPS measurements after 3 minutes of deposition are given in Table 1 , along with the $\mathrm{Ca} / \mathrm{P}$ and $\mathrm{O} / \mathrm{Ca}$ atomic ratios. In comparison, the theoretical $\mathrm{Ca} / \mathrm{P}$ ratios are $1.00,1.33,1.50,1.50$ and 1.67 for DCPD, OCP, ACP, TCP and HAp, respectively [30]. The theoretical O/Ca ratios are $6.00,3.125,3.00,2.67$ and 2.60 for these phases, respectively [30]. The measured $\mathrm{Ca} / \mathrm{P}$ and $\mathrm{O} / \mathrm{Ca}$ ratios for the bare gold surface and SAMs-covered surface gave values which match best those of $\mathrm{OCP}$. Yet, the measured $\mathrm{Ca} / \mathrm{P}$ atomic ratio obtained from conventional XPS analysis has been found to be always lower than the theoretical value for different calcium 
phosphates on the surface, thus preventing their unambiguous identification [30]. Hence, in order to identify the phases in a more definite way, the oxygen loss spectrum [25] was analyzed. The values of $\mathrm{O}(1 \mathrm{~s})_{\mathrm{II}} / \mathrm{O}(1 \mathrm{~s})$ are provided in Table 1 . In comparison, Lu et al. [30], Eliaz et al. [25] and Metoki et al. [31] measured the mean ratio of $\mathrm{O}(1 \mathrm{~s})_{\mathrm{II}} / \mathrm{O}(1 \mathrm{~s})$. Values of $0.072,0.065,0.053,0.037,0.020$ and 0.008 were obtained [30] for powders of TCP, HAp, OCP, dibasic calcium phosphate anhydrous (DCPA), DCPD, and monobasic calcium phosphate monohydrate (MCPM), respectively. Thus, the measured values reported in Table 1 for both the bare and the SAMs-covered surfaces fall within the values previously reported as typical of OCP. Similar analysis was carried out on bare and MAA-coated gold substrates after $\mathrm{CaP}$ electrodeposition for two hours; the results (not shown here) led to the same conclusions.

Further characterization of the effect of the functional groups on the deposition of $\mathrm{CaP}$ was accomplished by studying the morphological and structural changes of bare and SAM-coated Au samples after applying $-1.36 \mathrm{~V}$ for a longer deposition time of two hours (Fig. 6). While the bare sample shows large cracks and delamination, SAM-coated substrates show either finer cracks (ME) or apparently crack-free and more uniform (MAA) surfaces. While the samples coated with ME exhibit substantial cracks, they do not seem to peel-off.

Hence, it is evident that the presence of the thiol on the Au affects the deposition of $\mathrm{CaP}$; its functional groups (either $-\mathrm{OH}$ or $-\mathrm{CO}_{2} \mathrm{H}$ ) have a significant effect on the deposition. The gold surface after annealing is predominantly characterized by (111) face on which alkanethiols form a $\sqrt{3} \times \sqrt{ } 3 R 30^{\circ}$ adlayer [32,33]. On the other hand, OCP is characterized by a triclinic structure (space group P) [34]. If epitaxial matching is the dominant factor, we would not have found such significant differences between the OCP growth on both thiols. On the other hand, it is known that carboxylic acids enhance deposition of insoluble Ca salts due to the $\mathrm{Ca}^{2+}{ }_{-} \mathrm{COOH}$ favorable interactions. Hence, we believe that the results of MAA coated 
surface shown in figure 5, are due to the initial adsorption of $\mathrm{Ca}^{2+}$. Moreover, thiols can serve as a "molecular glue" (as has been shown and used in the past to promote the adhesion of gold onto glass [35]); they increase dramatically the adhesion of various materials onto gold. Hence, it is not surprising that the deposition of $\mathrm{CaP}$ onto $\mathrm{Au}$ in the presence of such a molecular glue results in less defects and lamination.

The CaP delamination from the bare surface can be explained by a mismatch in the crystallographic structures of $\mathrm{Au}$ and $\mathrm{CaP}$ and the high associated residual strains. If these strains cannot be accommodated by the film, dislocations are likely to be formed at the interface. Above a critical thickness, the increasing strains will lead to cracking [36]. As the mismatch on the bare surface is the largest, the cracking there is the most severe.

The thickness of the coating was measured in the ESEM on metallographic crosssections of samples coated with CaP for 2 hours (Supplementary Information, Fig. 2). The results are shown in Table 2. While the thickness of the CaP coating on ME-modified substrate is similar to that on bare $\mathrm{Au}$, the MAA-coated surface is covered by a much thinner layer (approximately half). This is not surprising considering the EQCM results that showed a much slower growth rate and a less efficient ED process on the MAA-coated Au surface.

In light of these results, a deposition mechanism is suggested, and presented in Scheme 1. While applying $-1.4 \mathrm{~V}$ reduction of water occurs on the electrode surface, producing hydroxyl ions. These react with the phosphoric acid in solution (the dominant species in this $\mathrm{pH}$ is $\mathrm{HPO}_{4}{ }^{2-}$ [16]) and deprotonate it. Then, the calcium in solution precipitates with the phosphate ions to create one of the $\mathrm{CaP}$ phases as seen in Fig. 1. These phases are thermodynamically more likely than the precipitation of calcium hydroxide, having a much higher $\mathrm{K}_{\mathrm{SP}}$ constant $\left(\mathrm{K}_{\mathrm{SP}, \mathrm{Ca}(\mathrm{OH}) 2}=5.5 \cdot 10^{-6}\right)$.

The ED on-top of MAA-covered substrates has significantly more side reactions, as seen in Fig. 2. Moreover, MAA-covered surfaces cause a 2D layered growth, as opposed to 
the 3D growth occurring on bare and ME-covered surfaces as seen if Fig 5. These two clues can give insight to the nucleation and growth of the coating. Kawashita et al. [37] suggested that the carboxylate groups facilitate the initial deposition of calcium ions from biomimetic solution, and that the attraction of calcium ions is an important initial step in calcium phosphate formation. More specifically, the role of the carboxylic acid, in the context of $\mathrm{CaP}$ electrodeposition, has been described before [38-40]. It was suggested that the presence of oxygen groups on the surface, enhances hydroxyl ions electrogeneration [40]. This can explain the elevation in cathodic current seen in Fig 2. Moreover, the carboxyl groups may be seen as active nucleation sites, attracting calcium ions, while hydroxyl ions diffuse outwards [39]. These conditions facilitate the formation of more homogenous crystals [39]. Therefore, the deprotonation of carboxylic terminal groups result in preferential sites to nucleate and $2 \mathrm{D}$ growth.

Moreover, EQCM data can shed more light on the steady-state growth of the MAAcovered surface. It is evident that while the initial period is similar to the other systems in growth rate, mass content and in phase, these parameters do not affect the steady-state deposition later on. Hence, the similarity and difference between the electrochemically assisted growth of $\mathrm{CaP}$ on bare $\mathrm{Au}$ and that coated with MAA and ME can be explained by assuming that although the same phase is formed, yet, the rate of its formation (whether 2D or 3D) is different and is affected by the monolayer beneath. The different rate of formation is presumably due to variations in grain size, plane orientation or real electrochemical surface area.

\section{Conclusions}

The growth of ED CaP on two different monolayers, 2-mercaptoacetic acid (MAA) and 2-mercaptoethanol (ME), was studied both at short and long time periods on well-defined 
evaporated gold surfaces. It was found that the end group of the monolayer has a major effect on the growth of the CaP coating. The carboxylic end group induced a higher cathodic current while requiring a higher overpotential for the reduction of water, which is a driving force for the deposition of $\mathrm{CaP}$. In the early stages of deposition, both the phase content and the mass gain are similar with or without SAMs. Nevertheless, a different growth mode is evident from different surface morphologies that are revealed by HR-SEM. While the deposition is slower and less efficient on a MAA-covered surface, mud-cracks are essentially eliminated thanks to lower crystallographic mismatch. The carboxylic acid is known to enhance the deposition of insoluble Ca-salts due to $\mathrm{Ca}^{2+}-\mathrm{COOH}$ favorable interaction. Hence, we suggest that MAA may facilitate $\mathrm{CaP}$ growth by attracting $\mathrm{Ca}^{2+}$ ions to the surface. Further test are presently carried out on titanium surfaces.

\section{Acknowledgement}

This study was financially supported by a Kamin grant 52694-5 from the Israel Ministry of Economy. The partial support by the Focal Technology Area through the Israel National Nanotechnology Initiative (INNI) is acknowledged. The authors thank Dr. Larisa Burstein from the Wolfson Applied Materials Research Centre at Tel-Aviv University for the XPS characterization service. The Harvey M. Krueger Family Centre for Nanoscience and Nanotechnology of the Hebrew University is acknowledged.

\section{References}

[1] N. Eliaz, T.M. Sridhar, Electrocrystallization of hydroxyapatite and its dependence on solution conditions, Cryst. Growth Des. 8(11) (2008) 3965.

[2] L. Zhao, M.D. Weir, H.H.K. Xu, Human umbilical cord stem cell encapsulation in calcium phosphate scaffolds for bone engineering, Biomaterials 31(14) (2010) 3848. 
[3] N. Eliaz, M. Eliyahu, Electrochemical processes of nucleation and growth of hydroxyapatite on titanium supported by real-time electrochemical atomic force microscopy, J Biomed. Mater. Res. A 80(3) (2007) 621.

[4] N. Eliaz, W. Kopelovitch, L. Burstein, E. Kobayashi, T. Hanawa, Electrochemical process of nucleation and growth of calcium phosphate on titanium supported by real-time quartz crystal microbalance measurements and X-ray photoelectron spectroscopy analysis, J Biomed. Mater. Res. A 89 (2009) 270.

[5] D. Gopi, J. Indira, L. Kavitha, A comparative study on the direct and pulsed current electrodeposition of hydroxyapatite coating on surgical grade stainless steel, Surf. Coat. Technol. 206 (2012) 2859.

[6] C. Montero-Ocampo, D. Villegas, L. Veleva, Controlled potential electrodeposition of calcium phosphate on Ti6A14V. J Electrochem. Soc. 152(10) (2005) C692.

[7] N. Eliaz, S. Shmueli, I. Shur, D. Benayahu, D. Aronov, G. Rosenman, The effect of surface treatment on the surface texture and contactangle of electrochemically deposited hydroxyapatite coatingand on its interaction with bone-forming cells, Acta Biomaterialia 5 (2009) 3178.

[8] Z. Grubač, M. Metikoš-Huković, R. Babić, Electrocrystallization, growth and characterization of calcium phosphate ceramics on magnesium alloys, Electrochimi. Acta 109 (2013) 694.

[9] M. Metikoš-Huković, R. Babic, Z. Grubač, M. Petravić, R. Peter, Potential assisted formation and characterization of hydroxyapatite coating on biodegradable magnesium alloy. J Electrochem. Soc. 160(10) (2013) H674.

[10] Q. Liu, J. Ding, F.K. Mante, S.L. Wunder, G.R. Baran, The role of surface functional groups in calcium phosphate nucleation on titanium foil: a self-assembled monolayer technique, Biomaterials 23 (2002) 3103.

[11] H. Li, W. Huang, Y. Zhang, M. Zhong, Biomimetic synthesis of enamel-like hydroxyapatite on self-assembled monolayers, Mater. Sci. Eng. 27(C) (2007) 756.

[12] P.J. Majewski, G. Allidi, Synthesis of hydroxyapatite on titanium coated with organic self-assembled monolayers, Mater. Sci. and Eng. A 420 (2006) 13.

[13] M. Tanahashi, T. Matsuda, Surface functional group dependence on apatite formation on self-assembled monolayers in a simulated body fluid, J. Biomed. Mater. Res. 34(3) (1997) 305 . 
[14] P. Li, K. Nakanishi, T. Kokubo, K. Groot, Induction and morphology of hydroxyapatite, precipitated from metastable simulated body fluids on sol-gel prepared silica, Biomaterials, 14(13) (1993) 963.

[15] A. Takeuchi, C. Ohtsuki, T. Miyazaki, M. Kamitakahara, S. Ogata, M. Yamazaki, Y. Furutani, H. Kinoshita, M. Tanihara, Heterogeneous nucleation of hydroxyapatite on protein: structural effect of silk sericin, J Royal. Soc. Interface 2 (2005) 373.

[16] N. Eliaz, Electrocrystallization of calcium phosphate. Isr. J Chem. 48 (2008) 159.

[17] J.C. Love, L.A. Estroff, J.K. Kriebel, R.G. Nuzzo, G.M. Whitesides, Self-assembled monolayers of thiolates on metals as a form of nanotechnology, Chem. Rev. 105 (2005) 1103.

[18] M. Byloos, H. Al-Maznai, M. Morin, Formation of a self-assembled monolayer via the electrospreading of physisorbed micelles of thiols, J Phys. Chem. B 103 (1999) 6554.

[19] K.P. Fears, S.E. Creager, R.A. Latour, Determination of the surface pK of carboxylicand amine- terminated alkanethiols using surface plasmon resonance spectroscopy, Langmuir 24(3) (2008) 837.

[20] D.V. Vezenov, A. Noy, L.F. Rozsnyai, C.M. Lieber, Force titrations and ionization state sensitive imaging of functional groups in aqueous solutions by chemical force microscopy, J. Am. Chem. Soc. 119 (1997) 2006.

[21] M.C. Leopold, J.A. Black, E.F. Bowden, Influence of gold topography on carboxylic acid terminated self-assembled monolayers, Langmuir 18 (2002) 978.

[22] Z. Dai, H.X. Ju, Effect of chain length on the surface properties of omega-carboxy alkanethiol self-assembled monolayers. Phys. Chem. Chem. Phys. 3(17) (2001) 3769.

[23] G.H. Lu, C.Y. Liu, H.Y. Zhao, W. Liu, L.P. Jiang, L.Y. Jiang, Determination of surface pKa of pure mercaptoacetic acid and 2-mercaptobenzothiazole mixed monolayers by impedance titration. Chinese Chem. Lett. 15 (7) (2004) 827.

[24] D.A. Brass, K.R. Shull, Membrane-enhanced surface acoustic wave analysis of grafted polymer brushes. J Appl. Phys. 103(7) (2008) 07351.

[25] N. Eliaz, W. Kopelovitch, L. Burstein, E. Kobayashi and T. Hanawa, Electrochemical processes of nucleation and growth of calcium phosphate on titanium supported by realtime quartz crystal microbalance measurements and X-ray photoelectron spectroscopy analysis, J Biomed. Mater. Res. Part A 89(1) (2009) 270-280.

[26] E.J. Martin, M.T. Mathew, K.R. Shull, Viscoelastic properties of electrochemically deposited protein/metal complexes, Langmuir 31 (2015) 4008.

[27] M. Uchida, H.M. Kim, T. Kokubo, S. Fujibayashi, T. Nakamura, Structural dependence 
of apatite formation on titania gels in a simulated body fluid, J. Biomed. Mater. Res. 64 (2003) 164.

[28] D.R. Desiraju, J.J. Vittal, A. Ramanan, Crystal Engineering - A textbook, World scientific publishing, Singapore, 2011.

[29] R.L. Karlinsey, K. Yi, C.W. Duhn, Nucleation and growth of apatite by a self-assembled polycrystalline bioceramic, Bioinsp. Biomim. 1 (2006) 12.

[30] H.B. Lu, C.T. Campbell, D.J. Graham, B.D. Ratner, Surface characterization of hydroxyapatite and related calcium phosphate by XPS and TOF-SIMS, Anal. Chem. 72 (2000) 2886.

[31] N. Metoki, L. Leifenberg-Kuznits, W. Kopelovich, L. Burstein, M. Gozin, N. Eliaz, Hydroxyapatite coating electrodeposited at near-physiological conditions, Material letters 119 (2014) 24.

[32] C.E.D. Chidsey, C.R. Bertozzi, T.M. Putvinski, A.M.J. Mujsce, Coadsorption of ferrocene-terminated and unsubstituted alkanethiols on gold - electroactive self-assembled monolayers, Am. Chem. Soc. 112(11) (1990) 4301.

[33] J.C. Love, L.A. Estroff, J.K. Kriebel, R.G. Nuzzo, G.M. Whitesides, Self-assembled monolayers of thiolates on metals as a form of nanotechnology, Chem. Rev. 105 (2005) 1103.

[34] Amjad Z (Ed.). Calcium Phosphate in Biological and Industrial Systems. Springer Science and Business Media, New York. 1998. p.2.

[35] C.A. Goss, D.H. Charych, M. Majda, Application of 3-Mercaptopropyl)trimethoxysliane as a molecular adhesive in the fabrication of vapor-deposited gold electrodes on glass substrates, Anal. Chem. 63(1) (1991) 85.

[36] O. Kresse, L. Truskinovsky, Lattice friction for crystalline defects: from dislocations to cracks, J Mech. Phys. Solids 52(11) (2004) 2521.

[37] M. Kawashita, M. Nakao, M. Minoda, H.M. Kim, T. Beppu, T. Miyamoto, T. Kokubo, T. Nakamura, Apatite-forming ability of carboxyl group-containing polymer gels in simulated body fluid. Biomaterials 24(2003)2477.

[38] H. Zanin, C.M.R. Rosa, N. Eliaz, P.W. May, F.R. Marciano, A.O. Lobo, Assisted deposition of nano-hydroxyapatite onto exfoliated carbon nanotube oxide scaffolds. R. Soc. Chem. 7(2015)10218.

[39] M.M. Zogbi Jr., E. Saito, H. Zanin, F.R. Marciano, A.O. Lobo, Hydrothermalelectrochemical synthesis of nano-hydroxyapatite crystals on superhydrophilic vertically aligned carbon nanotubes. Mater. Letters 132(2014)70. 
[40] A.O. Lobo, M.A.F. Corat, S.C. Ramos, J.T. Matsushima, A.E.C. Granato, C. PachecoSoares, E.J. Corat, Fast Preparation of hydroxyapatite/superhydrophilic vertically aligned multiwalled carbon nanotube composites for bioactive application. Langmuir 26(23)(2010)18308.

\section{Figures and Tables:}

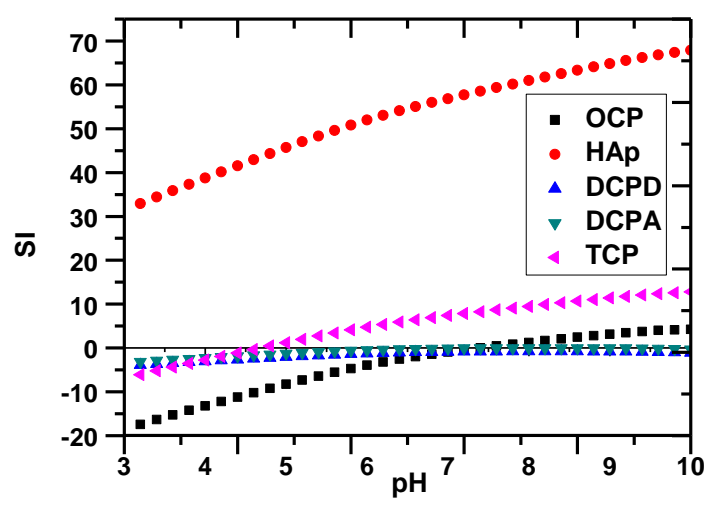

Figure 1. The dependence of the saturation indices (SI) of five calcium phosphates in the solution used for ED at $37^{\circ} \mathrm{C}$.

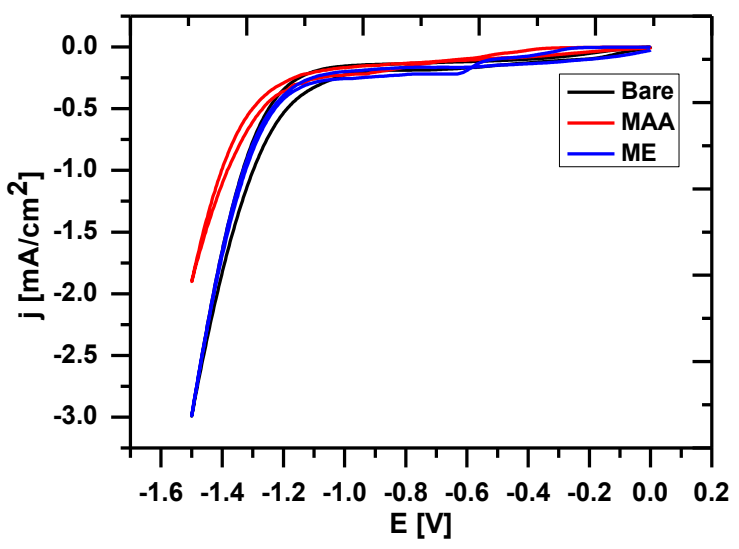

Figure 2. $\mathrm{CV}$ of both bare $\mathrm{Au}$ and $\mathrm{Au}$ coated with ME and MAA SAMs, as recorded in the solution used for ED at a scan rate of $10 \mathrm{mV} \mathrm{s}^{-1}$. 

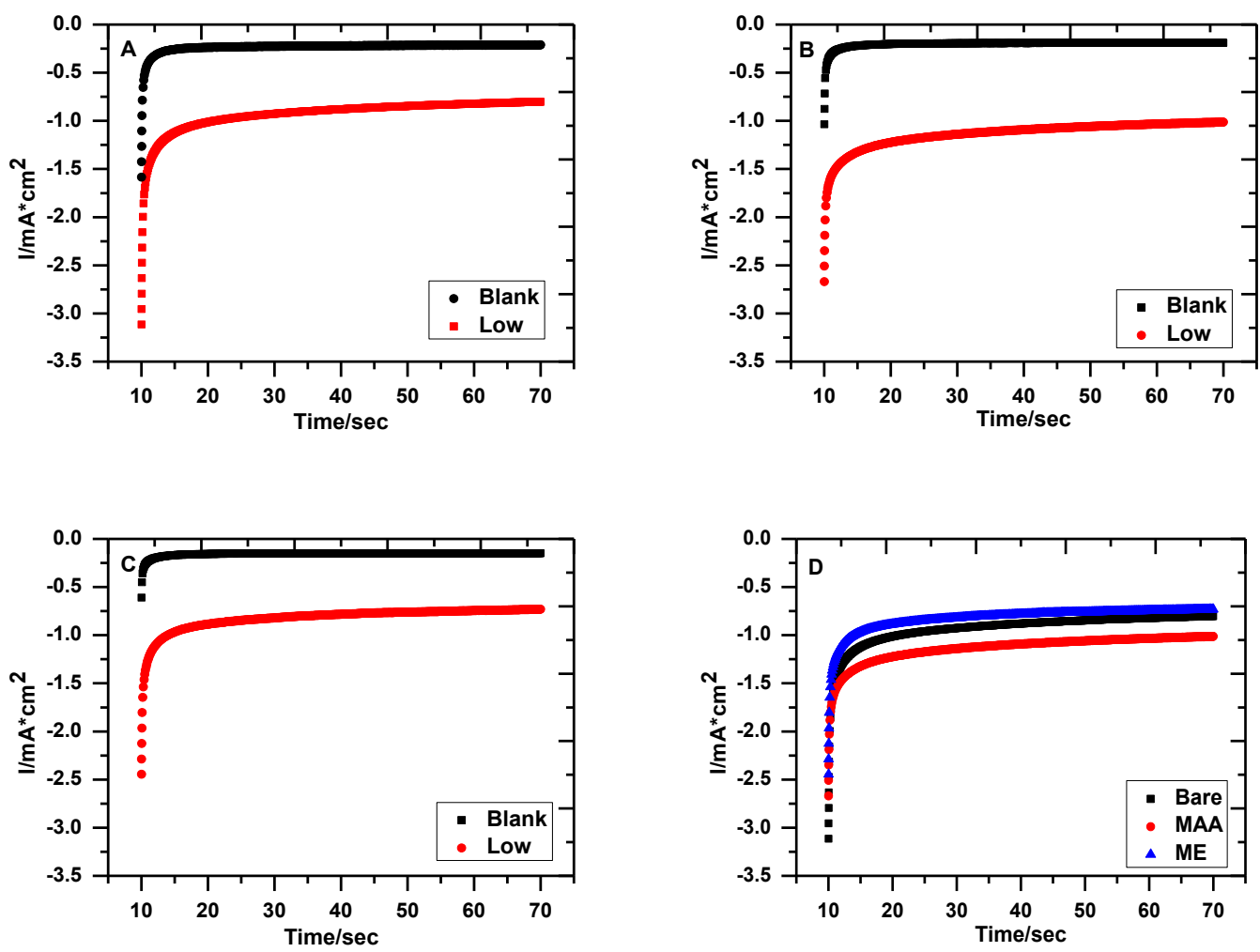

Figure 3. $\mathrm{CA}$ of bare $\mathrm{Au}(\mathrm{A})$ and $\mathrm{Au}$ coated with MAA (B) or ME (C) electrodes in a solution containing only $0.1 \mathrm{M} \mathrm{NaCl}$ (Blank), and in a solution containing in addition calcium and phosphate ions (Low). (D) Comparison between the different substrates in Low solution. The initial potentials were $0 \mathrm{~V} v s$. reference. 

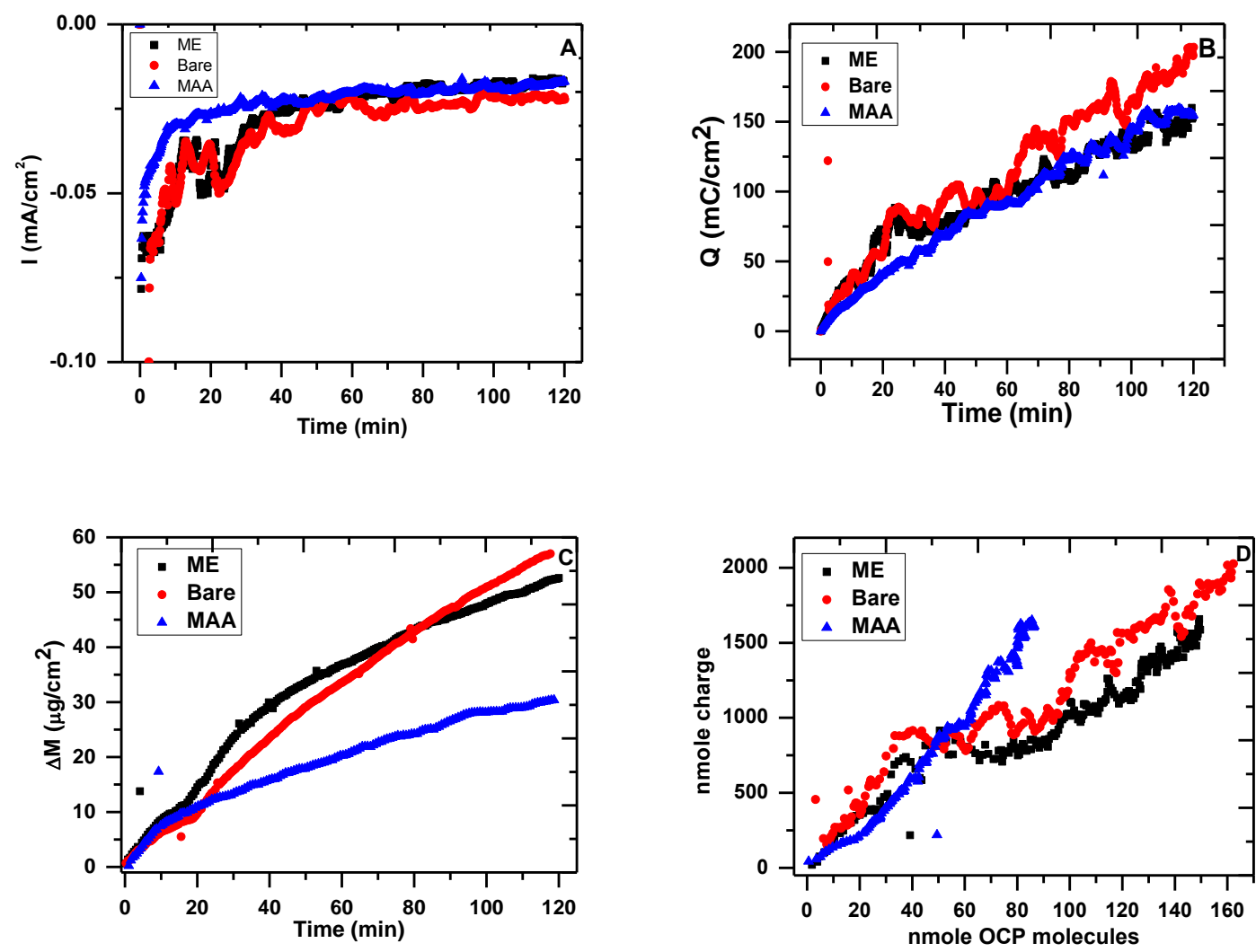

Figure 4. Current density (A), accumulated charge density (B), cumulative mass gain per surface area (C), and normalized mole of charge per mole of molecules (D). Data are drawn for both the bare and modified gold electrodes and are calculated based on the third harmonic in EQCM experiments. 

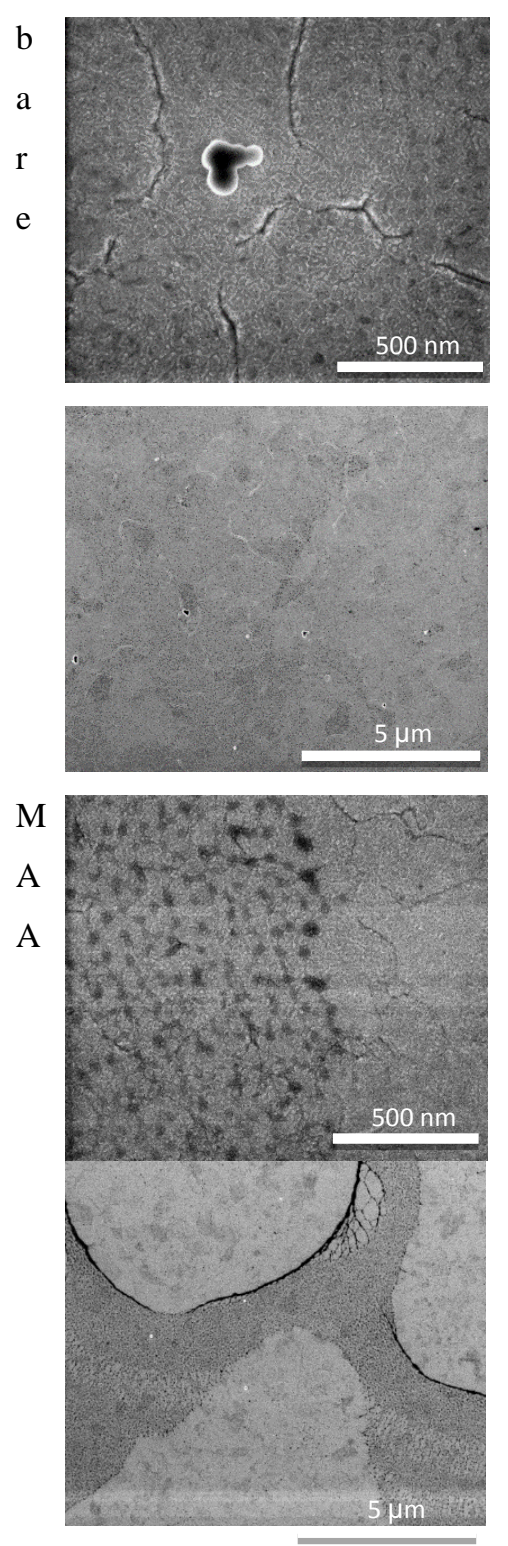

$30 \mathrm{sec}$
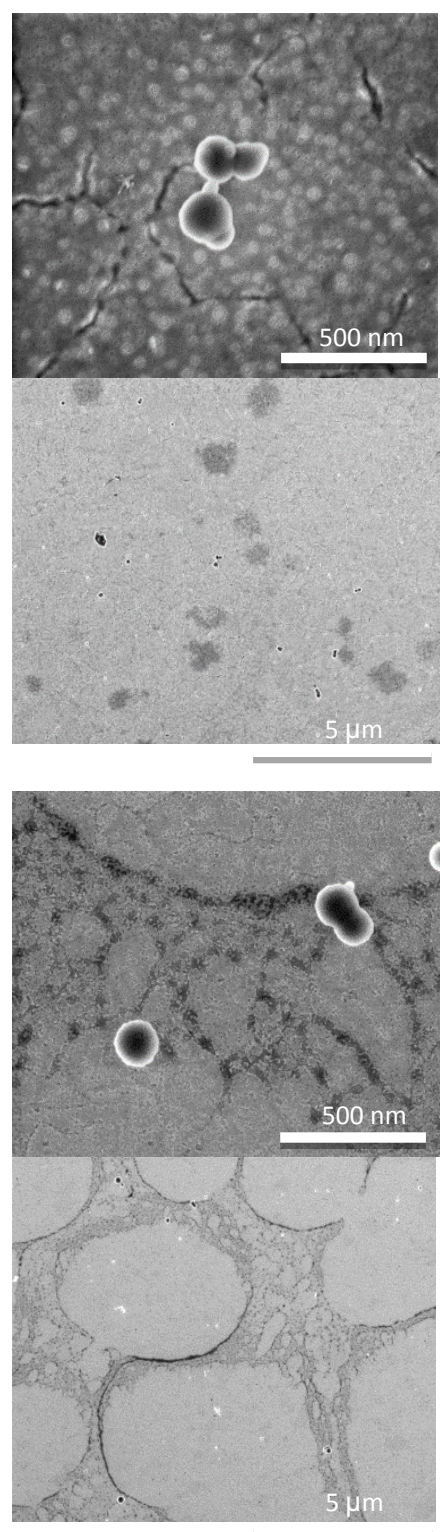

$1 \mathrm{~min}$
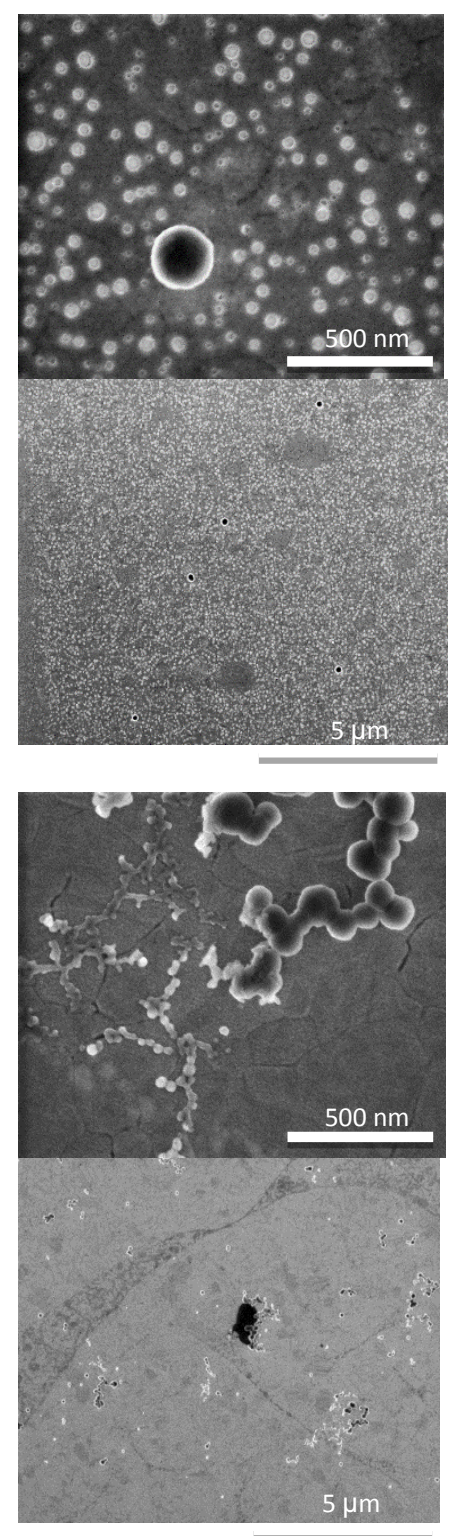

$2 \min$
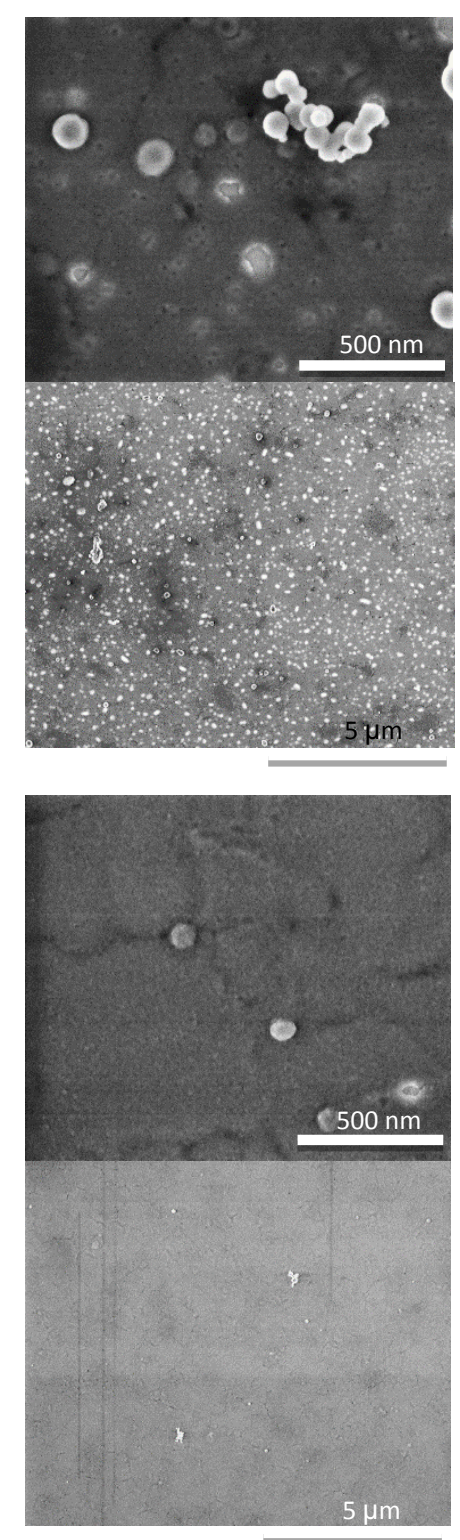

$3 \min$

Figure 5. ESEM images of CaP coating on bare (two upper rows) and MAA-coated gold (two lower rows) at different stages of growth. For each substrate, both low-magnification (lower) and high-magnification (upper) images are shown. 
Table 1 Chemical composition (at. \%) of the CaP coating after 3 min deposition on either bare gold or SAM-coated gold, as determined by high-resolution XPS.

\begin{tabular}{lcc}
\hline Element & Bare & $\mathrm{CO}_{2} \mathrm{H}(\mathrm{MAA}) \mathrm{SAM}$ \\
\hline $\mathrm{C}$ & $23.8 \pm 0.09$ & $23.1 \pm 0.14$ \\
$\mathrm{O}$ & $49.3 \pm 0.29$ & $49.6 \pm 0.13$ \\
$\mathrm{Ca}$ & $14.94 \pm 0.19$ & $15.04 \pm 0.06$ \\
$\mathrm{P}$ & $12 \pm 0.08$ & $12.24 \pm 0.14$ \\
$\mathrm{Ca} / \mathrm{P}$ & $1.24 \pm 0.02$ & $1.23 \pm 0.01$ \\
$\mathrm{O} / \mathrm{Ca}$ & $3.29 \pm 0.06$ & $3.30 \pm 0.02$ \\
$\mathrm{O}(1 \mathrm{~s})_{\mathrm{II}} / \mathrm{O}(1 \mathrm{~s})$ & $0.053 \pm 0.002$ & $0.054 \pm 0.001$ \\
\hline
\end{tabular}
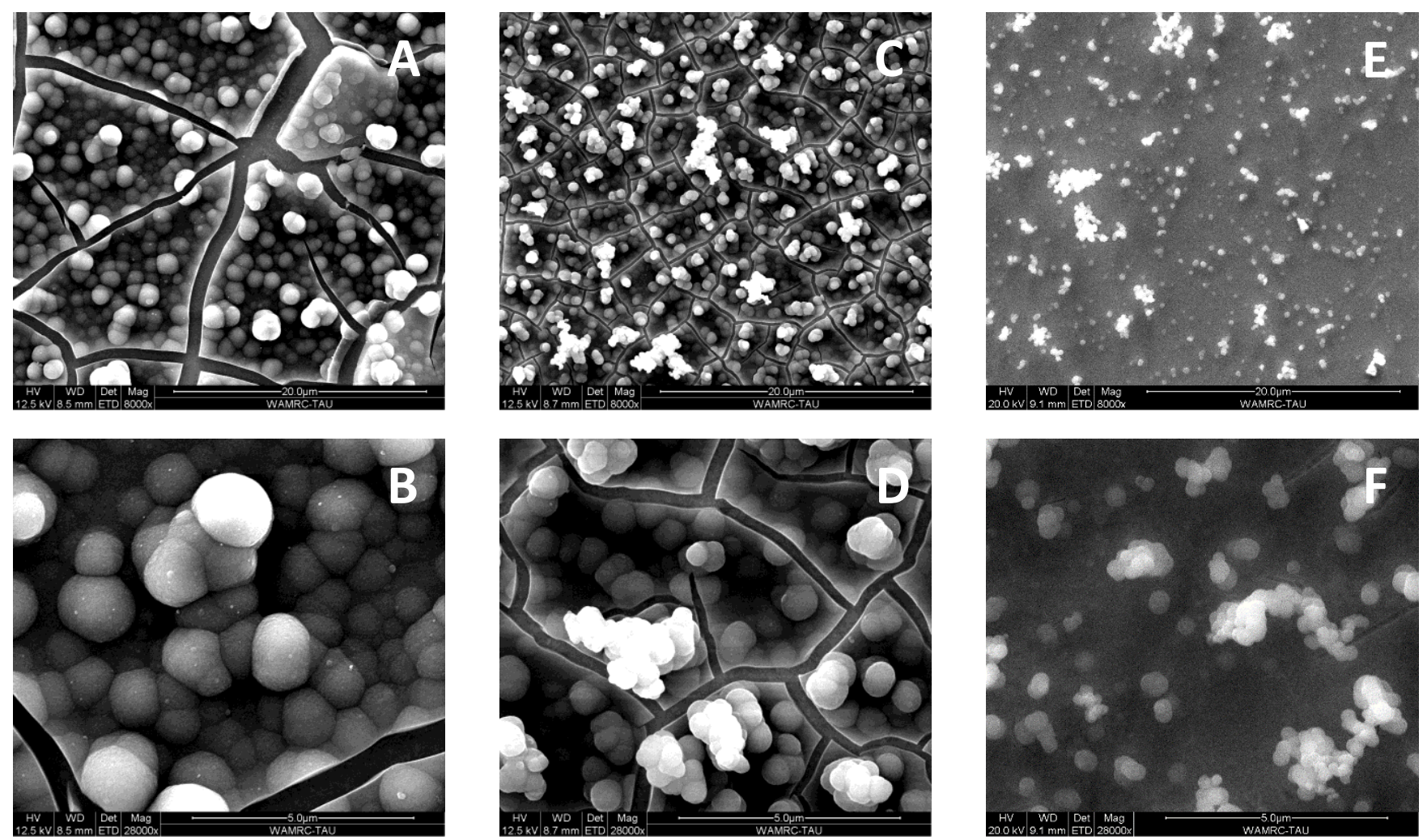

Figure 6. ESEM images of gold samples coated with CaP-coated for 2 hours. (A, B) Bare gold, (C, D) ME-coated gold, (E, F) MAA-coated gold. 
Table 2 Thickness measurements for CaP coating deposited for two hours on different gold substrates.

\begin{tabular}{ccc}
\hline & Mean $[\mu \mathrm{m}]$ & Standard deviation $[\mu \mathrm{m}]$ \\
\hline Bare & 1.11 & 0.47 \\
MAA & 0.49 & 0.17 \\
ME & 0.98 & 0.4 \\
\hline
\end{tabular}

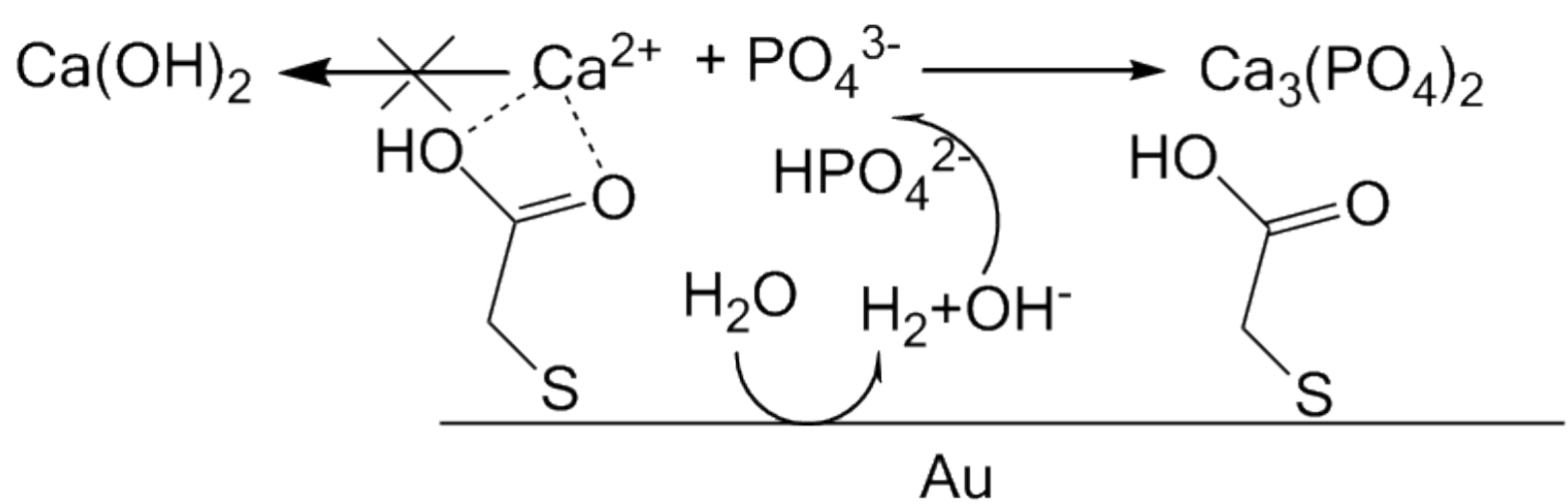

Scheme 1. CaP proposed deposition mechanism. 